\title{
A nonlocal model of phytoplankton aggregation
}

\author{
M. Adioui*, O. Arino, N. El Saadi ${ }^{1}$ \\ GEODES-IRD 32, Avenue Henri Varagnat, F-93143, Bondy cedex, France
}

Received 5 December 2003; accepted 8 November 2004

\begin{abstract}
The mechanisms of grouping and the models revolving around these problems truly impassioned many mathematicians. Our main goal in this paper is the development and analysis of an aggregation model of phytoplankton. The model is the continuum limit of an interacting particle model describing a "long-ranged" aggregation mechanism among particles. It consists of an integro-differential advection-diffusion equation, with a convolution term responsible for the agreggation process. The nonlinearity in the equation is homogeneous of degree one, which introduces several complications. We prove that the Cauchy problem associated to this model is well posed, i.e., there exists a unique global positive solution and it satisfies the principle of conservation of mass. Further, we establish the existence of nonuniform stationary solutions using the topological degree theory, namely Leray-Schauder's fixed point theorem. This asymptotic result agrees with our beliefs that nonlinear interactions at small scales can produce some aggregating patterns at large scales.
\end{abstract}

(C) 2005 Elsevier Ltd. All rights reserved.

Keywords: Phytoplankton aggregation; Interacting particles; Lagrangian model; Eulerian model; McKean-Vlasov interaction; Semigroups; Leray-Schauder's fixed point theorem

\section{Introduction}

Elucidating the underlying mechanisms responsible for the process of aggregate formation in phytoplankton at various spatial and time scales has been the goal of much experimental and theoretical research, and is to date the subject of a large body of biological and mathematical literature. Similar phenomena were observed in very varied fields:

\footnotetext{
* Corresponding author. Tel.: +3314802 55 83; fax: +33148473088.

E-mail address: adioui@bondy.ird.fr (M. Adioui).

${ }^{1}$ Partially supported by a PhD IRD grant (N/Ref:287976K/P240093).
} 
physics of the atmosphere, astrophysics, chemistry of colloids, hematology, epidemiology, phytoplanktonology, etc. This recently stimulated the mathematical study of equations from model groupings.

Phytoplankton cells have the ability of forming dispersed aggregates in the water column, which constitute the main food available to the early larval stages of many fish species, including the anchovy. At such stages, larvae are passive and can only eat the prey passing in very close vicinity. The best situation is when the larva is near a phytoplankton aggregate, while on the other hand larvae which stay far from aggregates are not likely to survive. Thus, being able to describe the distribution in numbers of phytoplankton aggregates of different sizes as well as locating them in the space turn out to be of utmost importance in connection with the study of fish recruitment.

Coagulation theory has more recently been applied to describe aggregation of marine particles [20] and specifically phytoplankton aggregation [10,13,24]. Many laboratory experiments [6,16,15], mesocosm experiments [4], and field observations [15-17] have all demonstrated that coagulation theory at times provides an accurate description of phytoplankton aggregate formation (see [14]). Aggregation by physical coagulation requires that primary particles collide by some physical process and stick together upon collision. Brownian motion, differences in sinking velocity between particles and fluid shear may all cause primary particles to collide.

However, studies of marine aggregates at small scales have emphasized biological mechanisms for their formation. That is, although planktonic organisms can be thought of as particles, the richness of biological responses makes the nature of their interactions more complex than the simple physical ones described by coagulation theory. Indeed, some planktonic species (algae, bacteria, dinoflagellates that are motile species of phytoplankton) have chemosensory abilities [7,26,25]: they can sense the chemical field generated by the presence of other particles. The dinoflagellates and more generally algae are known to leak organic matter into solution [19]. This leakage creates a zone around individual cells, the "phycosphere", where extracellular products exist in enhanced concentrations compared to the surrounding concentration [2]. The released products such as amino acids and sugar attract algae or bacteria present in a suitable neighborhood.

Our purpose in this paper is twofold: on the one hand, we propose a one-dimensional model that describes the aggregation behavior of phytoplankton on the vertical component of water column; on the other, we investigate the mathematical analysis of this model and explore its asymptotic behavior.

Our model consists of an integro-differential advection-diffusion equation with a convolution term, which is derived rigorously as the continuum limit from a large system of phytoplankton cells subject to random dispersal modeled as Brownian motions and mutual interactions allowing the particles' motions some dependence. It can be considered as an example of the class of models reviewed in [22]. The main feature of this model is that it captures the idea of the attraction interaction between organisms at small scales, due to the chemosensory behavior.

The paper is organized as follows. In Section 2, we describe thoroughly the model and in Section 3, we discuss its derivation from the corresponding interacting particle system. In Section 4, we show that the Cauchy problem associated to the aggregation model is well posed in a suitable function space. Indeed, we investigate the existence, uniqueness 
and positivity of solutions. We also prove that the value of the integral of the solution is a constant, so the solution remains a proportion density for all positive time. Global existence fails in many nonlinear situations. Here, it holds for nonnegative solutions. In Section 5, we study the existence of steady-state solutions. The complicated form of the model makes it difficult to determine stationary solutions straightforwardly. Nonetheless, by using the topological degree theory, we establish the existence of nonuniform steady-state solutions. Finally, we conclude with a brief discussion in Section 6.

\section{Model description}

The model we propose is given by the following partial differential equation:

$$
\begin{aligned}
\frac{\partial}{\partial t} u(x, t)= & d \frac{\partial^{2}}{\partial x^{2}} u(x, t)-\frac{\partial}{\partial x}\left(u(x, t) \Phi(x)\left[G * u^{0}(\cdot, t)\right](x)\right), \\
& \text { in } \Omega \times(0, \infty),
\end{aligned}
$$

where $\Omega=] 0, L$ [ is a bounded domain with smooth boundary $\partial \Omega$ in $\mathbb{R}, x$ is a one-dimensional coordinate, and $t$ is the time. This equation deals with temporal and spatial changes in the phytoplankton population density. $u(x, t)$ represents the spatio-temporal distribution density of phytoplankton on the vertical coordinate of water column, that is, $u(x, t) d A$ is the expected number of organisms in the sample area $d A$ surrounding the point $x$ at time $t$. Here, $\mathbb{R}$ represents the vertical axis oriented downward from the surface to the seabed and the interval $\Omega=] 0, L$ [ corresponds to the euphotic zone on the vertical component of water column. Generally, phytoplankton cells can survive only in the "euphotic zone", that is why we restrict our model to this domain. The depth of the euphotic zone varies widely depending on water clarity, latitude and season. It is often in the range of 50-100 m from the surface [9]. The diffusion term in (1) takes into account the spatial spread of the population with the coefficient of diffusion $d$; while the advection term describes the interaction mechanisms among particles via the velocity $G * u^{0}$. The latter has the form of convolution [21], i.e.,

$$
\left[G * u^{0}(., t)\right](x)=\int_{\mathbb{R}} G\left(x-x^{\prime}\right) u^{0}\left(x^{\prime}, t\right) \mathrm{d} x^{\prime},
$$

where

$$
u^{0}(x)= \begin{cases}u(x), & 0<x<L \\ 0, & x \leqslant 0 \text { or } x \geqslant L\end{cases}
$$

and the kernel $G$ is given by

$$
G(x)=-\left[-|x|^{2}+\left(r_{0}+r_{1}\right)|x|-r_{0} r_{1}\right] \operatorname{sign}(x) 1_{\left[r_{0}, r_{1}\right]}(|x|) .
$$

$\left(G * u^{0}\right)(x)$ describes the velocity induced at the site $x$ by the net effects of all individuals at various sites $x^{\prime}$. It is clear from (2) that $G\left(x-x^{\prime}\right)$ leads to the attraction of the particle at position $x$ to the one at $x^{\prime}$, as a function of the distance between the two particles. To give a biological explanation of the interaction mechanism among particles, we make two assumptions: (a) the nonuniform concentration fields around organisms, (b) organisms 
considered as having chemosensory abilities and hence some "knowledge" of their neighbors whereabouts and modifying their motion accordingly. More precisely, if we consider a phytoplankton particle located in $x$, the extracellular products released by this particle form a high concentration zone around $x$, on a radius of length $r_{0}\left(r_{0}>0\right)$, which attracts all particles located at positions $x^{\prime}$ such that the distance between $x$ and $x^{\prime}$ lies in the interval $\left[r_{0}, r_{1}\right]\left(r_{1}>r_{0}\right)$. Beyond $r_{1}$, particles cannot perceive the difference in concentration because they are sensory limited $[3,11,12]$. Hence, they are not attracted. The sign of $G\left(x-x^{\prime}\right)$ changes according to whether $x^{\prime}$ is above or below $x$ : $G$ behaves as a gradient, that is, a particle is attracted to the region of high density. Boundary conditions are imposed at the surface and at $L$ :

$$
\frac{\partial}{\partial x} u(x, t)=0, \quad \text { on } \partial \Omega \times \mathbb{R}^{+}
$$

and the initial condition is

$$
u(x, 0)=u_{0}(x) \geqslant 0, \quad \text { in } \Omega .
$$

We also assume that $u_{0}$ is a density distribution, that is,

$$
\int_{0}^{L} u_{0}(x) \mathrm{d} x=1
$$

The function $\Phi$ in the advection term is an extra term to the dynamics of the phytoplankton population. It has been set in the model only for mathematical convenience, that is, to eliminate the advection term at the bounds 0 and $L$ since condition (3) is not sufficient. $\Phi$ is chosen such that

$$
\Phi \in H_{0}^{1}(\Omega), \quad \sup \Phi \subset[\delta, L-\delta], \quad \delta \text { sufficiently small, }
$$

where sup $\Phi$ denotes the support of function $\Phi$.

The normalization condition (5) is connected to the fact that the system is conservative. We will not consider growth terms, and focus exclusively on nonlinear and nonlocal transport properties of the population.

\section{Model derivation}

As already mentioned, the continuum density given by Eq. (1) is derived by a limiting process from the empirical distribution associated with a large number of particles.

The interacting particles model in $\mathbb{R}$ corresponding to (1) is

$$
\mathrm{d} X_{N}^{k}(t)=\frac{1}{N} \sum_{j=1}^{N} G\left(X_{N}^{k}-X_{N}^{j}\right) \mathrm{d} t+\sqrt{2 d} \mathrm{~d} B^{k}(t), \quad k=1, \ldots, N,
$$

where $X_{N}^{k}(t) \in \mathbb{R}$ is the position of particle $k$ at time $t$ and $B^{k}(t), k=1, \ldots, N$ are independent $\mathbb{R}$-valued Brownian motions. The dispersion term expresses the vertical diffusion 
of phytoplankton cells in water which is similar to molecular diffusion [3] and $d$ is the coefficient of diffusion. The drift term indicates the attractive force exerted on the $k$ th particle due to the interaction of this particle with all others in the population.

Following [22], we may give the collective behavior of the system in terms of the stochastic measure-valued process $\left\{X_{N}(t)\right\}_{t \in \mathbb{R}^{+}}$, where

$$
X_{N}(t)=\frac{1}{N} \sum_{k=1}^{N} \delta_{X_{N}^{k}(t)}
$$

with $\delta_{X_{N}^{k}(t)}$ being the Dirac measure at the location $X_{N}^{k}(t)$.

The system of SDE (7) can be expressed in terms of the empirical distribution (8):

$$
d X_{N}^{k}(t)=\left(G * X_{N}(t)\right)\left(X_{N}^{k}(t)\right) \mathrm{d} t+\sqrt{2 d} d B^{k}(t), \quad k=1, \ldots, N .
$$

It is clear that we deal with a McKean-Vlasov interaction or "long-ranged" interaction (the range of interaction is independent of $N$ ). Since $G$ is a bounded Lipschitz continuous function, the propagation of chaos theory shows that the empirical process $\left\{X_{N}(t)\right\}_{t \in \mathbb{R}^{+}}$ converges in law, as $N$ goes to infinity, to a deterministic measure-valued process $\{X(t)\}_{t \in \mathbb{R}^{+}}$ and this limiting process admits for any $t \in \mathbb{R}^{+}$a density $u(x, t)$ with respect to the Lebesgue measure on $\mathbb{R}$ (see [28] for a review; [8]). Consequently, by using the same techniques as in [22], we obtain that $u(x, t)$ satisfies:

$$
\frac{\partial}{\partial t} u(x, t)=d \frac{\partial^{2}}{\partial x^{2}} u(x, t)-\frac{\partial}{\partial x}(u(x, t)[G * u(., t)](x)), \quad \text { in } \mathbb{R} \times(0, \infty) .
$$

We recognize that (9) is similar to Eq. (1) but without the term $\Phi$.

As mentioned in [22, p. 10], in order to complete rigorously the proof of the convergence from the Lagrangian model to the evolution equation (1) for the spatial density of the deterministic process $\{X(t)\}_{t \in \mathbb{R}^{+}}$, one has to provide existence and uniqueness for the density $u(x, t)$ satisfying (1). These problems will be investigated in the next section.

\section{Existence, uniqueness and positivity}

\subsection{Abstract formulation}

The approach we shall adopt in our analysis of (1)-(4) is to reformulate the associated initial-value problem as an abstract Cauchy problem, which can be treated using the theory of semigroups of operators. We obtain a quasi-linear problem with nonlinearities in the first-order term:

$$
\left\{\begin{array}{l}
\frac{\mathrm{d}}{\mathrm{d} t} u(t)=A u(t)-B\left[u(t) g_{(\Phi, G)}(u(t))\right] \\
u(0)=u_{0}
\end{array}\right.
$$


in which $u(t)$ is used for $u(., t)$. The operator $A: D(A) \subset X:=L^{2}(\Omega) \rightarrow X$ is defined by

$$
\begin{aligned}
& A w=d \frac{\mathrm{d}^{2} w}{\mathrm{~d} x^{2}}, \\
& D(A)=\left\{w \in H^{2}(\Omega): w_{\mid \partial \Omega}^{\prime}=0\right\}
\end{aligned}
$$

and the operator $B: D(B) \subset X \rightarrow X$ by

$$
\begin{aligned}
& B w=\frac{\mathrm{d}}{\mathrm{d} x} w, \\
& \mathscr{D}(B)=H^{1}(\Omega) .
\end{aligned}
$$

$H^{1}(\Omega)$ and $H^{2}(\Omega)$ denote usual Sobolev functions spaces. We will denote by $\langle$,$\rangle and \|$.$\| ,$ respectively, the scalar product and the norm in $X$. The operator $A$ commutes with $B$ and they are related by the following formula:

$$
\langle B u, d B u\rangle=-\langle u, A u\rangle, \quad \forall u \in \mathscr{D}(A) .
$$

We endow $D(B)$ with the graph norm

$$
|x|_{\mathscr{D}(B)}=\|x\|_{B}+\|B x\|, \quad \forall x \in D(B) .
$$

The main existence result will be derived using successive approximations in a space of continuous functions from some suitable interval $\left[0, t_{0}\right]$ (where $t_{0}>0$ will be chosen later on) into $D(B)$. On occasion, we will use the notation $Y=C\left(\left[0, t_{0}\right], D(B)\right)$. The operator $g_{(\Phi, G)}$ is defined as follows:

$$
g_{(\Phi, G)}(\varphi)(x)=\Phi(x)[G * \varphi](x)=\Phi(x) \int_{\mathbb{R}} G(x-y) \varphi(y) \mathrm{d} y .
$$

By straightforward consequence of standard calculations, we can establish that $g_{(\Phi, G)}$ : $D(B) \rightarrow D(B)$ continuously, so there exists a constant $\delta$, so that

$$
\left|g_{(\Phi, G)}(\varphi)\right|_{\mathscr{D}(B)} \leqslant \delta|\varphi|_{\mathscr{D}(B)}, \quad \forall \varphi \in \mathscr{D}(B) .
$$

Note also that $G * u^{0}$ is uniformly bounded. Hence, $g_{(\Phi, G)} u$ is uniformly bounded. As a result of Hölder's inequality, we obtain

$$
\left|g_{(\Phi, G)} u\right|_{\infty} \leqslant \sqrt{L}|G|_{\infty}|\Phi|_{\infty}\|u\|, \quad \forall u \in D(B) .
$$

On the other hand, we have

$$
\left|B g_{(\Phi, G)} u\right|_{\infty} \leqslant \sqrt{L}|G|_{\infty} \max \left(|\Phi|_{\infty},|B \Phi|_{\infty}\right)|u|_{\mathscr{D}(B)}, \quad \forall u \in D(B) .
$$

The differential equation (10) may not have a strong solution. Thus we solve it in integrated form by using the variation of constants formula

$$
u(t)=T(t) u_{0}-\int_{0}^{t} T(t-s) B\left[u(s) g_{(\Phi, G)}(u(s))\right] \mathrm{d} s .
$$

We recall that a solution of (16) is called a mild solution of the differential equation (10). 
Proposition 1. The operator A defined by (11) is the generator of an analytic semigroup of contractions in $X,(T(t))_{t \geqslant 0}$, compact for $t>0$. The restrictions $T(t) / \mathscr{D}(B)$ send $\mathscr{D}(B)$ into itself and are uniformly bounded in $\mathscr{D}(B)$ (that is, there exists $C_{1} \geqslant 0$, such that, $\left|T(t)_{/ \mathscr{D}(B)}\right|_{D(B)} \leqslant C_{1}$, for $\left.t \geqslant 0\right)$.

Proof. The proof is similar to the one in [1].

\subsection{Local existence of solutions}

This subsection is concerned with the local existence of solutions for problem (10). The main problem we faced here was the fact that $B$ is not a fractional power of $(-A)$; this complicates the treatment of the problem.

For this purpose, we start by establishing some useful estimates.

Lemma 2. (1) There exists a constant $M$, such that, for all $u, v \in \mathscr{D}(B)$, we have

$$
\left\|B\left[u_{(\Phi, G)}(u)\right]-B\left[v g_{(\Phi, G)}(v)\right]\right\| \leqslant M \max \left(|u|_{\mathscr{D}(B)},|v|_{\mathscr{D}(B)}\right)|u-v|_{\mathscr{D}(B)} .
$$

(2) There exists a positive constant $Q$, such that, for all $u \in \mathscr{D}(B)$, it holds that

$$
\left\|B\left[u g_{(\Phi, G)}(u)\right]\right\| \leqslant Q|u|_{\mathscr{D}(B)}\|u\| .
$$

(3) There exists a positive constant $C$, such that, for all $u \in X$, it holds that

$$
\|B T(t) u\| \leqslant \frac{C}{\sqrt{t}}\|u\|, \quad \forall t>0 .
$$

Proof. For the claim, the proof is the same as the one given in [1, Lemma 2.1].

We can now state the main theorem of this subsection.

Theorem 3. For every $R>0$, there exists $t_{0}>0, t_{0}=t_{0}(R)$, such that, for each $u_{0} \in$ $\mathscr{B}_{\mathscr{D}(B)}(R)$, (i.e., the ball of radius $R$ centered at 0 of $\left.\mathscr{D}(B)\right)$, the Cauchy problem $(10)$ has a unique mild solution $u$ defined on the interval $\left[0, t_{0}\right]$. Moreover, the map $u_{0} \rightarrow u$ is Lipschitz continuous from $\mathscr{B}_{\mathscr{D}(B)}(R)$ into $Y$.

Proof. The proof is presented in Appendix (A 1).

\subsection{Global existence}

The result proved in the previous theorem is valid on a local time interval, but it can be extended to arbitrarily large times. In fact, we have the following result.

Theorem 4. For every $u_{0} \in \mathscr{D}(B)$, there exists a unique global positive solution of Eq. (10) and it satisfies the principle of conservation of mass.

A proof of Theorem 4, which is very technical and independent of the rest of the paper, is presented in Appendix (A 2). 


\subsection{Regularity}

The following result describes the regularity of a mild solution of (10).

Theorem 5. For every $u_{0} \in \mathscr{D}(A)$, the mild solution of Eq. (10) is a classical solution, i.e. $u$ is continuous on $[0, \infty)$, continuously differentiable on $(0, \infty), u(t) \in D(A)$ for $t \in(0, \infty)$ and (10) is satisfied on $[0, \infty)$.

The proof is based on the arguments used in the proof of Theorem 2.4 in [1], so we just sketch its main points. In fact, if we show that the function

$$
f(t)=B\left[u(t) g_{(\Phi, G)}(u(t))\right]
$$

is locally Hölder continuous from $(0, \infty)$ into $\mathscr{D}(B)$; then it follows from the theory of nonhomogeneous linear equations (see, [23, Corollary 3.3]) that the mild solution of (10) is a classical solution. That $f$ is locally Hölder continuous on $(0, \infty)$ will follow if we prove that the map $t \rightarrow u(t)$ is locally Hölder continuous, from $(0, \infty)$ to $\mathscr{D}(B)$.

\section{Steady-state solutions}

\subsection{Fixed point problem}

We consider the problem made up of (1)-(3)-(4)-(6)-(5) and we look for stationary solutions, i.e., for solutions of the form $u(x, t)=u(x), \forall t$.

Such solutions must satisfy the system:

$$
d \frac{\mathrm{d}}{\mathrm{d} x} u(x)-u(x) \Phi(x)\left[G * u^{0}(.)\right](x)=\gamma,
$$

where $\gamma$ is a constant to be determined, $x \in \Omega$, with boundary conditions

$$
\frac{\partial u}{\partial x}_{\mid x=0, L}=0 .
$$

Using the boundary condition (19) together with (6), we deduce that

$$
\begin{aligned}
& \gamma=0, \\
& u(x) \geqslant 0 \quad \text { in } \Omega \text { and } \int_{0}^{L} u(x) \mathrm{d} x=1 .
\end{aligned}
$$

Let us introduce the following operator $\mathscr{F}$ defined by

$$
\mathscr{F}(u)(x)=\int_{0}^{x} \frac{1}{d} \Phi(y)\left[G * u^{0}(.)\right](y) \mathrm{d} y .
$$


In terms of $\mathscr{F}(u),(18)$ can be written as

$$
\frac{\mathrm{d}}{\mathrm{d} x} u(x)-u(x) \frac{\mathrm{d}}{\mathrm{d} x} \mathscr{F}\left(u^{0}\right)(x)=0 .
$$

By integration by parts and using the boundary condition, we arrive at

$$
u(x)=u(0) \exp \left(\mathscr{F}\left(u^{0}\right)(x)\right),
$$

which, together with the normalization condition,

$$
\int_{0}^{L} u(x) \mathrm{d} x=1
$$

leads to

$$
u(x)=\frac{\exp \left(\mathscr{F}\left(u^{0}\right)(x)\right)}{\int_{0}^{L} \exp \left(\mathscr{F}\left(u^{0}\right)(s)\right) \mathrm{d} s}=\operatorname{def} \mathscr{H}(u)(x) .
$$

Then, from (23), the search for steady-state solutions is equivalent to finding fixed points of $\mathscr{H}$ :

$$
u=\mathscr{H}(u) .
$$

Remark 6. It is visible that the uniform distribution $\bar{u}=1 / L$ does not satisfy (24). Therefore, if a steady-state solution exists it could not be the uniform distribution.

\subsection{Steady-state solutions}

In this part, we deal with the problem of determining steady-state proportion densities. For this, we will use the topological degree theory, namely we use Leray-Schauder's fixed point Theorem. To know more about this approach, we can refer, for example, to [5]. Let us start with an observation connected to the operator $\mathscr{H}$.

Using some standard algebra, one has:

$$
\int_{0}^{z} \frac{1}{d} \Phi(x)\left[G * u^{0}(.)\right](x) \mathrm{d} x=\int_{0}^{L} u(y) k(z, y) \mathrm{d} y,
$$

with

$$
k(z, y)=\int_{0}^{z} \frac{1}{d} \Phi(x) G(x-y) \mathrm{d} x .
$$

Using this observation, the operator $\mathscr{H}$, defined by formula (23) can be written as

$$
\mathscr{H}(u)(z)=\frac{\exp \left[\int_{0}^{L} u(y) k(z, y) \mathrm{d} y\right]}{\int_{0}^{L} \exp \left[\int_{0}^{L} u(x) k(z, x) \mathrm{d} x\right] \mathrm{d} z} .
$$

We introduce the following operator $\mathscr{H}_{\theta}$ defined by

$$
\mathscr{H}_{\theta}(u)(z)=\frac{\exp \left[\int_{0}^{L} u(y) k(\theta z, y) \mathrm{d} y\right]}{\int_{0}^{L} \exp \left[\int_{0}^{L} u(x) k(\theta z, x) \mathrm{d} x\right] \mathrm{d} z},
$$


where $\theta$ is a real parameter, $\theta \in[0,1]$ and let us look for steady-state solutions of the problem

$$
u=\mathscr{H}_{\theta}(u)
$$

Evidently, if we define $\bar{u}=1 / L$, it holds that $\mathscr{H}_{0}(\bar{u})=\bar{u}$.

We are now in a position to draw the main result of the existence of steady-state solutions:

Theorem 7. The fixed point problem (24) has a solution.

A proof of Theorem 7 is presented in Appendix A.3.

\section{Conclusion}

The aim of this work was to develop a mathematical model of aggregation in phytoplankton that helps to understand the mechanisms underlying spatio-temporal pattern formation. Here, the grouping phenomenon is a consequence of social behavior and is due to the nonlinear interactions between phytoplankton particles. The model has been rigorously (not heuristically as for many continuum models) derived by a limiting process from the empirical distribution associated to an interacting system of particles. It describes the evolution of the mean-field spatial density of phytoplankton population on the vertical water column by a deterministic nonlinear partial differential equation of the advection-diffusion type. To provide the existence and uniqueness for the solutions of this equation, we have proved that the Cauchy problem (10) associated to this model is well posed in $\mathscr{D}(B)$. Solutions are fixed points of strict contractions and initial values in $\mathscr{D}(A)$ yield classical solutions. We have also proved the conservation of mass of phytoplankton, that is, the solution of our problem remains a proportion density for all positive time. Further, we explored the asymptotic behavior of the model, namely the existence of steady-state solutions. The complicated form of the model does not allow to obtain steady-state solutions (not even a trivial solution) by the usual method as in [1]. Nevertheless, using the topological degree theory, more precisely, Leray-Schauder's fixed point theorem, we established the existence of a steady-state solution. We do not know about the stability of this solution but we stress on the fact that the latter could not be a uniform distribution, in any case. This is what we have expected: the nonlinear interactions between organisms candidates for heterogeneity and emergence of patterns on the vertical water column. Simulations are necessary to complete the study.

\section{Acknowledgements}

The authors would like to thank Prof. Tanya Kastova and unknown referees for their comments, which allowed to improve the original manuscript. The authors also thank Prof. Rafael Bravo, Dr. Daniela Morale and Prof. Jean-Pierre Treuil for numerous useful discussions. 


\section{Appendix A.}

\section{A.1. Proof of Theorem 3}

The proof is very similar to that of Theorem 2.1 in [1]. Therefore we only give an outline. As mentioned earlier, the approach to be taken in the proof is based on the method of successive approximations. Let $u_{0} \in \mathscr{B}_{\mathscr{D}(B)}(R)$ and define a sequence $\left(u_{n}\right)_{n \geqslant 1}$ by

$$
u_{n+1}(t)=T(t) u_{0}-\int_{0}^{t} T(t-s) B\left[u_{n}(s) g_{(\Phi, G)}\left(u_{n}(s)\right)\right] \mathrm{d} s .
$$

Assume that the sequence $u_{n}$ is bounded in $C\left(\left[0, t_{0}\right], X\right)$, namely, that there exist $t_{0}>0$ and $\gamma$ to be determined later on, such that

$$
\left|u_{n}(t)\right|_{\mathscr{D}(B)} \leqslant \gamma R, \quad \forall n \geqslant 1 \quad \text { and } \quad \forall t \in\left[0, t_{0}\right] .
$$

Using Lemma 2, we show that

$$
\begin{aligned}
& \left|u_{n+1}(t)-u_{n}(t)\right|_{\mathscr{D}(B)} \\
& \quad \leqslant[M \gamma R(2 C \sqrt{t}+t)] \sup _{0 \leqslant s \leqslant t}\left|u_{n}(s)-u_{n-1}(s)\right|_{\mathscr{D}(B)}, \quad \forall t \geqslant 0 \text { and } n \geqslant 1 .
\end{aligned}
$$

Then, by choosing $t_{0}>0$ small enough so that

$$
\left[M \gamma R\left(2 C \sqrt{t_{0}}+t_{0}\right)\right]<\frac{1}{2}
$$

the sequence $\left(u_{n+1}(t)-u_{n}(t)\right)$ is the general term of an absolutely convergent series in $Y$. To complete the proof of the theorem, we have to show that the sequence $u_{n}(t)$ remains bounded in $\mathscr{D}(B)$.

Following [1], we obtain

$$
\left|u_{n+1}(t)\right|_{\mathscr{D}(B)} \leqslant C_{1} R+\gamma R\left[2 C \sqrt{t_{0}}+t_{0}\right]\left(\sqrt{L}|\Phi|_{\infty}|G|_{\infty} \gamma R+L\right), \quad \forall t \in\left[0, t_{0}\right] .
$$

Therefore, by choosing $t_{0}>0$ so that (29) holds and

$$
\left[2 C \sqrt{t_{0}}+t_{0}\right]\left(\sqrt{L}|G|_{\infty}|\Phi|_{\infty} \gamma R+L\right)<\frac{1}{2}
$$

we arrive at

$$
\left|u_{n+1}(t)\right|_{\mathscr{D}(B)} \leqslant C_{1} R+\frac{1}{2} \gamma R, \quad \text { for } t \leqslant t_{0} .
$$

This inequality will be extended to $n+1$ if we can choose both $\gamma$ large enough for

$$
C_{1} R+\frac{1}{2} \gamma R \leqslant \gamma R \quad \text { (which holds as soon as } \gamma \geqslant 2 C_{1} \text { ) }
$$

and $t_{0}>0$ such that (29) and (30) hold which can always be accomplished, once $\gamma$ has been chosen. So, assuming $\gamma$ and $t_{0}$ have been chosen as indicated, the first claim of the proof is proved.

For the uniqueness of the solution $u$ and the Lipshitz continuity of the map $u_{0} \rightarrow u$ in the ball $\mathscr{B}_{\mathscr{D}(B)}(R)$, the proof is a straightforward consequence of standard calculations used in the proof of Theorem 2.1 in [1]. This completes the proof of Theorem 3. 


\section{A.2. Proof of Theorem 4}

In order to prove global existence (i.e., the fact that the solutions are defined on the whole of $t>0$ ), we begin by showing the following result which is very crucial in continuation of solutions.

Theorem A.1. For every initial data $u_{0} \in \mathscr{D}(B)$, the abstract Cauchy problem (10) has a unique mild solution on a maximal interval of existence $\left[0, t_{\max }\left[\right.\right.$. If $t_{\max }<\infty$ then

$$
\lim _{t \rightarrow t_{\max }} \sup |u(t)|_{\mathscr{D}(B)}=\infty
$$

The proof of this result is similar to the proof of Theorem 2.2 in [1]. Now, we will show the boundedness of the solution $u(t)$ in the $\mathscr{D}(B)$ norm. This property, together with Theorem A.1, implies that $t_{\max }=\infty$. But, prior to this, we prove that $(C P)$ preserves positivity, which will be needed in the a priori estimates of the solutions.

Theorem A.2. Eqs. (1)-(4) preserve positivity, that is $u_{0} \geqslant 0$ implies that $u(x, t) \geqslant 0$ for all $t \geqslant 0$.

Proof. Let $u(., t)$ be a solution of system (1)-(4) and $u^{-}(x, t)=\max (0,-u(x, t))$ in $x \in \Omega$ for each $t \geqslant 0$.

Now multiplying (take inner products) both sides of equality (1) by $u^{-}(x, t)$ and integrating, we obtain

$$
\begin{aligned}
\int_{\Omega} u^{-}(x, t) \frac{\partial}{\partial t} u(x, t) \mathrm{d} x= & d \int_{\Omega} u^{-}(x, t) \frac{\partial^{2}}{\partial x^{2}} u(x, t) \mathrm{d} x \\
& +\int_{\Omega}\left(u(x, t) \Phi(x)\left[G * u^{0}(., t)\right](x)\right) \frac{\partial}{\partial x} u^{-}(x, t) \mathrm{d} x .
\end{aligned}
$$

Using the obvious identity

$$
-\left|u^{-}(x, t)\right|^{2}=u^{-}(x, t) u(x, t),
$$

we obtain

$$
\begin{aligned}
-\frac{1}{2} \frac{\mathrm{d}}{\mathrm{d} t} \int_{\Omega}\left|u^{-}(x, t)\right|^{2} \mathrm{~d} x= & d \int_{\Omega}\left[\frac{\partial}{\partial x} u^{-}(x, t)\right]^{2} \mathrm{~d} x \\
& -\int_{\Omega}\left(u^{-}(x, t) \Phi(x)\left[G * u^{0}(., t)\right](x)\right) \frac{\partial}{\partial x} u^{-}(x, t) \mathrm{d} x .
\end{aligned}
$$

Via the identity

$$
a b \leqslant \varepsilon a^{2}+\frac{b^{2}}{\varepsilon},
$$


combined with (14), we have

$$
\begin{aligned}
& \int_{\Omega}\left(u^{-}(., t) \Phi(x)\left[G * u^{0}(., t)\right](x)\right) \frac{\partial}{\partial x} u^{-}(x, t) \mathrm{d} x \\
& \quad \leqslant \frac{1}{\varepsilon} L_{0}\left(1+2 \sqrt{L}\left\|u^{-}(., t)\right\|\right)^{2}\left\|u^{-}(., t)\right\|^{2}+\varepsilon\left\|\frac{\partial}{\partial x} u^{-}(., t)\right\|^{2},
\end{aligned}
$$

(for some constant $L_{0}$, independent on $u^{-}(., t)$ ). Therefore,

$$
\begin{aligned}
\frac{\mathrm{d}}{\mathrm{d} t} \int_{\Omega}\left|u^{-}(x, t)\right|^{2} \mathrm{~d} x \leqslant & \frac{2}{\varepsilon} L_{0}\left(1+2 \sqrt{L}\left\|u^{-}(., t)\right\|\right)^{2}\left\|u^{-}(., t)\right\|^{2} \\
& +2(\varepsilon-d)\left\|\frac{\partial}{\partial x} u^{-}(., t)\right\|^{2} .
\end{aligned}
$$

By choosing $\varepsilon>0$ small enough, we can eliminate the second term of the right-hand side of (31). So, we arrive at the differential inequality

$$
\frac{\mathrm{d}}{\mathrm{d} t}\left\|u^{-}(., t)\right\|^{2} \leqslant L^{\prime}\left\|u^{-}(., t)\right\|^{2}\left(1+\left\|u^{-}(., t)\right\|\right)^{2} .
$$

( $L^{\prime}$ is a constant independent on $u^{-}(., t)$ ).

Following [1], we obtain

$$
\left\|u^{-}(., t)\right\|=0 \quad \text { for all } t \geqslant 0,
$$

that is, $u(., t) \geqslant 0$, for all $t \geqslant 0$.

Using the above result, we can give an estimate of the solutions as follows:

Proposition A.3. There exists a function $\left.\left.K: \mathbb{R}^{+} \rightarrow\right] 0,+\infty\right]$, nonincreasing, such that, if $u(., t)$ is a solution of $(C P)$ with $u_{0} \in \mathscr{D}(B)$ and $u_{0} \geqslant 0$; then, it holds that $|u(t)|_{\mathscr{D}(B)} \leqslant K_{1}$ $\left(\left\|u_{0}\right\|\right)\left|u_{0}\right|_{\mathscr{D}(B)}$, for all $t \in\left[0, K\left(\left\|u_{0}\right\|\right)\right]$, where $K_{1}(x)=2\left[C_{1}+\exp \left(L_{1} K(x)\right)\right]$.

The proof is based on the arguments used in the proof of Proposition 2.2 in [1], so we just sketch its main points. The claim is made in two steps. First, we show that the solution is bounded in $X$ norm on each bounded time interval. Then, we prove boundedness in the $\mathscr{D}(B)$ norm, using the result for the $X$ norm.

Finally, integrating both sides of Eq. (1) on 0 to $L$, and using (3) and (6), we establish conservation of mass of phytoplankton, that is,

$$
\int_{\Omega} u(x, t) \mathrm{d} x=\int_{\Omega} u_{0}(x) \mathrm{d} x=1 \text { for all } t \geqslant 0 .
$$

This completes the proof of Theorem 4.

\section{A.3. Proof of Theorem 7}

The operator $\mathscr{H}_{\theta}$ defined by (26) is continuous and compact, for each $\theta$. Hence the condition $\gamma$-condensing is satisfied. Such a measure $\gamma$ of noncompactness is a map from 
the family of bounded subsets into $\mathbb{R}^{+}$such that, in particular, $\gamma(B)=0$ iff $B$ is relatively compact (see [5, Definition 9.1]). Furthermore, one can see that $I-\mathscr{H}_{\theta}$ has no fixed point on $\partial \Gamma$, where

$$
\Gamma=\mathscr{B}_{L_{+}^{1}(\Omega)}(0,2)=\left\{u \in L_{+}^{1}(\Omega), \int u(x) \mathrm{d} x<2\right\} .
$$

Using an observation made above, that is, $\bar{u}$ is the only fixed point of (27), we have

$$
\operatorname{Deg}\left(I-\mathscr{H}_{0}, \Gamma, 0\right)=\operatorname{Deg}(I, \Gamma, 0)=1,
$$

in which $\operatorname{Deg}\left(I-\mathscr{H}_{0}, \Gamma, 0\right)$ denotes the topological degree for the map $I-\mathscr{H}_{0}$. Then according to Leray-Schauder's fixed point Theorem (see [5, Theorem 18.1]), it follows that

$$
\operatorname{Deg}\left(I-\mathscr{H}_{\theta}, \Gamma, 0\right)=1, \quad \forall \theta \in[0,1] .
$$

So, we conclude that the fixed point equation for $\mathscr{H}_{\theta}$ has a solution for each $\theta \in[0,1]$. Thus, problem (27) has a solution for all $\theta \in[0,1]$ and in particular, for $\theta=1$, which completes the proof of Theorem 7.

\section{References}

[1] M. Adioui, O. Arino, W.V. Smith, J. P Treuil, A mathematical analysis of a fish school model, J. Differential Equations 188 (2003) 406-446.

[2] W. Bell, R. Mitchell, Chemotactic and growth responses of marine bacteria to algal extracellular products, Biol. Bull. 143 (1972) 265-277.

[3] H.C. Berg, Random Walks in Biology, Princeton University Press, Princeton, p. 152.

[4] H.G. Dam, D.T. Drapeau, Coagulation efficiency, organic-matter glues and the dynamics of particles during a phytoplankton bloom in a mesocosm study, Deep-Sea Res. 42 (1995) 111-123.

[5] K. Deimling, Non-linear Functional Analysis, Springer, Berlin, New York, 1985.

[6] D.T. Drapeau, H.G. Dam, G. Grenier, An improved flocculator design for use in particle aggregation experiments, Limnol. Oceanogr. 39 (1994) 723-729.

[7] W.K. Fitt, Chemosensory responses of the symbiotic dinoflagellate Symbiodinium microadriatica (Dinophycae), J. Phycol. 21 (1985) 62-67.

[8] J. Gärtner, On the Mckean-Vlasov limit for interacting diffusions, Math. Nachr. 137 (1988) 197-248.

[9] S. Ghosal, M. Rogers, A. Wray, The turbulent life of phytoplankton, Proceedings of the Summer Programm, Center for Turbulence Research, 2000.

[10] P.S. Hill, Reconciling aggregation theory with observed vertical fluxes following phytoplankton blooms, J. Geophys. Res. 97 (1992) 2295-2308.

[11] G.A. Jackson, Simulating chemosensory responses of marine microorganisms, Limnol. Oceanogr. 32 (6) (1987) 1253-1266.

[12] G.A. Jackson, Simulation of bacterial attraction and adhesion to falling particles in an aquatic environment, Limnol. Oceanogr. 34 (3) (1989) 514-530.

[13] G.A. Jackson, A model of the formation of marine algal flocks by physical coagulation processes, Deep-Sea Res. 37 (1990) 1197-1211.

[14] T. Kiørboe, Formation and fate of marine snow: small-scale processes with large-scale implications, Sci. Mar. 655 (Suppl. 2) (2001) 57-71.

[15] T. Kiørboe, J.L.S. Hansen, A.L. Alldredge, G.A. Jackson, et al., Sedimentation of phytoplankton during a diatom bloom: rates and mechanisms, J. Mar. Res. 54 (1996) 1123-1148.

[16] T. Kiørboe, C. Lundsgaard, M. Olesen, J.L.S. Hansen, Aggregation and sedimentation processes during a spring phytoplankton bloom: a field experiment to test coagulation theory, J. Mar. Res. 52 (1994) 297-323. 
[17] T. Kiørboe, P. Tiselius, B. Mitchell-Innes, J.L.S. Hansen, A.W. Visser, X. Mari, Intensive aggregate formation with low vertical flux during an upwelling-induced diatom bloom, Limnol. Oceanogr. 43 (1998) 104-116.

[19] T.H. Mague, E.D. Friberg, J. Hughes, I. Morris, Extracellular release of carbon by marine phytoplankton; a physiological approach, Limnol. Oceanogr. 25 (1980) 262-279.

[20] I.N. McCave, Size spectra and aggregation of suspended particles in the deep ocean, Deep-Sea Res. 31 (1984) 329-352.

[21] A. Mogilner, E. Keshet, A nonlocal model for a swarm, J. Math. Biol. 38 (1999) 534-570.

[22] D. Morale, et al., An interacting particle system modelling aggregation behavior: from individuals to populations, J. Math. Biol. 2004, in press.

[23] A. Pazy, Semigroups of linear operators and applications to partial differential equations, Applied Mathematical Sciences, vol. 119, Springer, New York, 1983.

[24] U. Riebesel, D.A. Wolf-Gladrow, The relationship between physical aggregation of phytoplankton and particle flux: a numerical model, Deep-Sea Res. 39 (1992) 1085-1102.

[25] H.J. Spero, Chemosensory capabilities in the phagotrophic dinoflagellate Gymnodinium fungiforme, J. Phycol. 21 (1985) 181-184.

[26] H.J. Spero, M. Morée, Phagotrophic feeding and its importance to the life cycle of the holozoic dinoflagellate, Gymnodinium fungiforme, J. Phycol. 17 (1981) 43-51.

[28] A.S. Sznitman, Topics in propagation of chaos, in: P.L. Hennequin (Ed.), Ecole d'Eté de Probabilités de Saint-FlourXIX, Lecture Notes in Mathematics, 1989, vol. 1464, Springer, Berlin, Heidelberg, New York, 1991, pp. 165-251. 